\title{
M. Zenker: Monographs in human genetics Vol. 17, Noonan syndrome and related disorders—a matter of deregulated Ras signalling
}

\author{
Karger, 2009, HB, 167 p., EUROS 141.50. ISBN:978-3-8055-8653-5
}

\author{
Sally J. Davies
}

Published online: 3 April 2009

(C) Springer-Verlag 2009

One of the most exciting recent developments within dysmorphology has been the molecular unravelling of the RasMAPK pathway and the increased understanding of the clinical presentation of the mutations that alter the functioning of the molecules within the signalling cascade. There has long been argument about the clinical overlap between the common clinical condition Noonan syndrome and the rarer Cardio-Facio-Cutaneous, Costello and Leopard syndromes. The discovery that activating mutations in PTPN11, KRAS, SOS1, RAF1 and MEK1 cause Noonan syndrome and reduced function mutations in PTPN11 and increased function mutations in RAF1 give Leopard syndrome raises great interest in the interaction of the genes within the Ras-MAPK pathway. The further discovery that mutations in BRAF, MEK1 and 2 and KRAS within the pathway cause Cardio-Facio-Cutaneous syndrome and HRAS mutations, Costello syndrome, endorses the phenotypical similarities but emphasise the fascinating interaction of these genes.

This volume of Monographs in Human Genetics is a welcome addition to the series. Martin Zenker has invited experts within this clinical and molecular field to contribute chapters, including a History of Noonan syndrome and related disorders by Dr. Noonan herself. The format of each chapter follows a pattern which allows each chapter to be read independently and provides a well referenced review of the current knowledge. This does lead to some repetition if one is reading the volume as a whole but that is not a significant criticism.

Each of the clinical chapters is a well written review of the clinical features and related complications of the

S. J. Davies ( $\square)$

Institute of Medical Genetics, University Hospital of Wales,

Cardiff, CF 14 4XN, UK

e-mail: Daviessj8@ cardiff.ac.uk relevant disorder with good clinical images and illustrations. It is important to be aware of the related malignancy risk associated with particular conditions and mutations within specific genes. The genotype-phenotype correlations are very important for clinicians as well as the molecular scientists undertaking research into the pathway. This volume will be of relevance and interest to both clinicians and scientists alike.

The chapters related to the molecular genetics of each condition are well organised and emphasise the contribution of each gene within the overall signalling cascade. Specific somatic mutations which are known to be oncogenic are delineated together with any correlations with known reported malignancy in Noonan syndrome and the related conditions.

Further informative chapters are devoted to the endocrine regulation of growth and short stature in Noonan syndrome, the heart in Ras-MAPK pathway disorders and myeloproliferative disease and cancer in persons with Noonan syndrome and related disorders. The intriguing clinical overlap between Noonan syndrome and Neurofibromatosis type 1 has long taxed clinicians and scientists alike. This is reviewed with relevance to the interaction between the relevant genes within the pathway. The last two chapters deal with the knowledge gained from animal models and the intriguing possibility of reduction in activity of the RasMAPK pathway as a treatment modality for cancers associated with somatic mutations in the pathway as well as for Noonan syndrome and related conditions in the future.

This volume is a timely addition to the literature and provides a welcome review of the dysregulation of the Ras-MAPK pathway in Noonan syndrome and the related syndromes. It is very easy to navigate with clear chapters and references. I can certainly recommend it to anyone who wishes to update themselves on this fascinating, evolving story. 